\title{
An Evaluation of a Flight Deck Interval Management Algorithm including Delayed Target Trajectories
}

\author{
Kurt A. Swieringa ${ }^{1}$, Matthew C. Underwood ${ }^{2}$, and Bryan Barmore ${ }^{3}$ \\ NASA Langley Research Center, Hampton, VA, 23606 \\ and \\ Robert D. Leonard ${ }^{4}$ \\ Virginia Commonwealth University, Richmond, VA, 23284
}

\begin{abstract}
NASA's first Air Traffic Management (ATM) Technology Demonstration (ATD-1) was created to facilitate the transition of mature air traffic management technologies from the laboratory to operational use. The technologies selected for demonstration are the Traffic Management Advisor with Terminal Metering (TMA-TM), which provides precise timebased scheduling in the terminal airspace; Controller Managed Spacing (CMS), which provides controllers with decision support tools enabling precise schedule conformance; and Interval Management (IM), which consists of flight deck automation that enables aircraft to achieve or maintain precise in-trail spacing. During high demand operations, TMA-TM may produce a schedule and corresponding aircraft trajectories that include delay to ensure that a particular aircraft will be properly spaced from other aircraft at each schedule waypoint. These delayed trajectories are not communicated to the automation onboard the aircraft, forcing the IM aircraft to use the published speeds to estimate the target aircraft's estimated time of arrival. As a result, the aircraft performing IM operations may follow an aircraft whose TMA-TM generated trajectories have substantial speed deviations from the speeds expected by the spacing algorithm. Previous spacing algorithms were not designed to handle this magnitude of uncertainty. A simulation was conducted to examine a modified spacing algorithm with the ability to follow aircraft flying delayed trajectories. The simulation investigated the use of the new spacing algorithm with various delayed speed profiles and wind conditions, as well as several other variables designed to simulate real-life variability. The results and conclusions of this study indicate that the new spacing algorithm generally exhibits good performance; however, some types of target aircraft speed profiles can cause the spacing algorithm to command less than optimal speed control behavior.
\end{abstract}

Nomenclature

$A D S-B=$ Automatic Dependent Surveillance-Broadcast

ANOVA $=$ Analysis of Variance

$A R T C C=$ Air Route Traffic Control Center

$A S T A R=$ Airborne Spacing for Terminal Area Routes

ATC $=$ Air Traffic Controller/Control

ATD-1 = Air Traffic Management Technology Demonstration-1

ATM = Air Traffic Management

ATOS = Airspace and Traffic Operations simulation

CMS $\quad=$ Controller Managed Spacing

${ }^{1}$ Research Aerospace Engineer, Crew Systems and Aviation Operations Branch, NASA Langley Research Center, Mail Stop 152, Hampton, VA 23681-2199, AIAA Member.

${ }^{2}$ Research Aerospace Engineer, Crew Systems and Aviation Operations Branch, NASA Langley Research Center, Mail Stop 152, Hampton, VA 23681-2199, AIAA Member.

${ }^{3}$ Senior Research Engineer, Crew Systems and Aviation Operations Branch, NASA Langley Research Center, Mail Stop 152, Hampton, VA 23681-2199.

${ }^{4}$ Graduate Research Assistant, Department of Statistical Sciences and Operations Research, Virginia Commonwealth University, Richmond, VA 


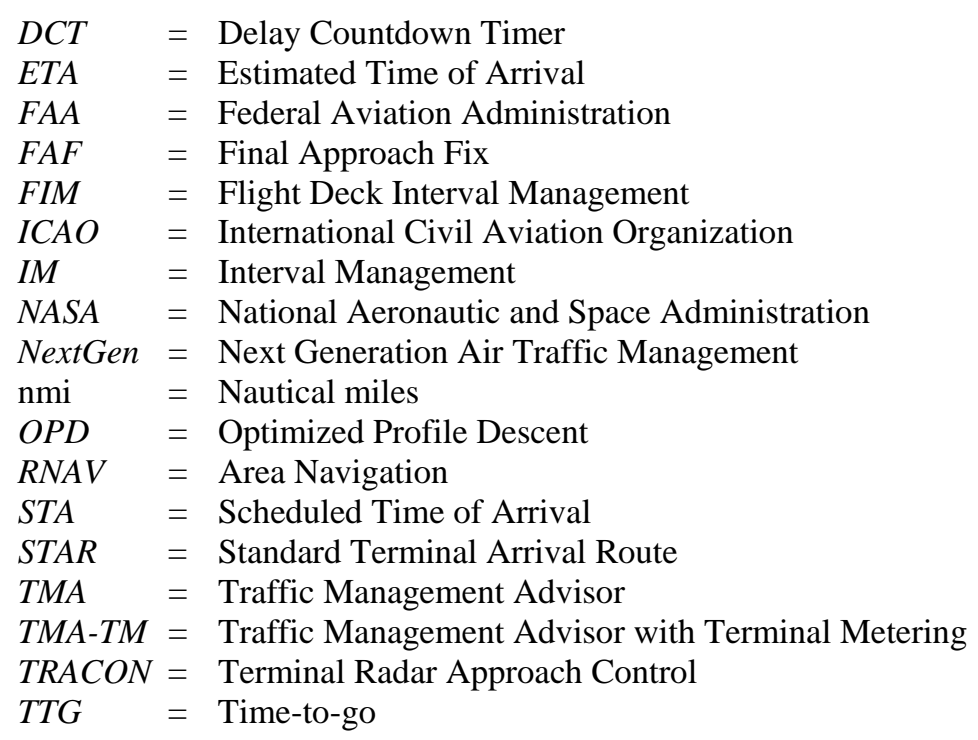

\section{Introduction}

Over the next twenty years, the Federal Aviation Administration (FAA) is predicting a substantial increase in the number of revenue passenger miles flown. ${ }^{1}$ This increase could strain the current air traffic system if no improvements are made. To help increase the efficiency of arrivals, fuel efficient procedures such as Optimized Profile Descents (OPDs) are being implemented at a number of sites. However, these procedures are often not used during periods of peak traffic due to the lack of precision scheduling and spacing tools, resulting in sub-optimal arrival operations. ${ }^{2}$ Researchers at the National Aeronautics and Space Administration (NASA) have developed technologies that enable the use of efficient arrival procedures during high demand operations.

The goal of NASA's first Air Traffic Management (ATM) Technology Demonstration (ATD-1) is to accelerate the implementation of mature NASA technologies, enabling aircraft to use speed control to remain close to their OPDs during periods of peak traffic. Three NASA technologies were selected to be integrated to achieve this goal: the Traffic Management Advisor with Terminal Metering (TMA-TM), which provides precise time-based scheduling in the terminal airspace; Controller Managed Spacing (CMS), which provides controllers with decision support tools that enable precise schedule conformance; and Interval Management (IM), which consists of flight deck automation that enables aircraft to achieve or maintain precise in-trail spacing behind the preceding aircraft. ${ }^{3}$ These technologies extend precision scheduling into the terminal area and provide pilots and controllers with control mechanisms that increase schedule conformance and achieve precise in-trail spacing.

During high demand operations, TMA-TM can produce a schedule and corresponding aircraft trajectories that include a substantial amount of delay to ensure a particular aircraft is properly spaced at each of the schedule waypoints. Since advanced air-ground communication will not be available in the airspace during the ATD-1 demonstration, these delayed trajectories cannot be communicated to the aircraft. As a result, aircraft conducting IM operations, hereinafter referred to as IM aircraft, use the published speeds to compute Estimated Times of arrival (ETAs) for the IM aircraft and the preceding aircraft, hereinafter referred to as the target aircraft. When the target aircraft flies a delayed trajectory, the ETA calculated by the IM aircraft is inaccurate, forcing the IM aircraft to tactically resolve the delay concurrently with the target aircraft. Previous spacing algorithms were not designed to compensate for large target aircraft speed deviations caused by the delayed trajectories.

This paper describes a modification to the existing NASA spacing algorithm designed to improve performance when following aircraft with delayed trajectories and a batch computer simulation that evaluated the new spacing algorithm. The first section discusses problems that were encountered when conducting IM operations with delayed target aircraft and a proposed algorithm to alleviate those problems. The following sections describe the design, results, and conclusions of a computer simulation that examined the new spacing algorithm. 


\section{Background}

\section{A. Technology Description}

TMA-TM is an extension of the Traffic Management Advisor (TMA) that adds terminal metering.,5 TMA-TM uses the predicted trajectory of each aircraft along its projected OPD to compute its ETAs to a series of scheduling waypoints. The schedule is then deconflicted at each of the scheduling waypoints by delaying aircraft until they are properly spaced. At a particular distance or time from the airport, known as the freeze horizon, TMA-TM locks the Scheduled Times of Arrival (STAs) at each of the metering waypoints. Both the STAs and ETAs computed by TMA-TM, along with the amount of delay an aircraft has to absorb, can be shown on timelines to center and terminal controllers.

CMS is a suite of decision support tools that provides terminal controllers with advisories that they can use to achieve the schedule provided by TMA-TM. ${ }^{6,7}$ The CMS tools consist of three pieces of information: early/late indicators, slot markers, and speed advisories. The early/late indicator is displayed in each aircraft's full data block and enables controllers to quickly assess an individual aircraft's schedule conformance. Slot markers are graphical representations of the schedule that indicate an aircraft's current scheduled location. Speed advisories are displayed on an aircraft's full data block, and provide controllers with recommended speeds to achieve schedule conformance.

IM is a flight deck tool that helps pilots achieve or maintain precise in-trail spacing behind a preceding aircraft. The avionics used to conduct an IM operation, referred to as the Flight deck Interval Management (FIM) avionics, consist of a spacing algorithm onboard the aircraft that uses data provided by controllers along with Automatic Dependent Surveillance-Broadcast (ADS-B) data from the target aircraft to compute IM commanded speeds that the flight crew follows to achieve precise in-trail spacing at a designated achieve-by point. ${ }^{8,9}$ NASA Langley has been developing an implementation of IM that uses a trajectory-based spacing algorithm that enables aircraft to perform IM operations when their target aircraft is on a different route. The procedure for conducting an IM operation begins when ATC provides an appropriately equipped aircraft with an IM clearance, which includes the target aircraft's callsign, a spacing goal computed by TMA-TM, and the target aircraft's route. After receiving the clearance, the flight crew loads it into onboard avionics and follows the speeds provided by the IM algorithm to precisely achieve the spacing interval set by TMA-TM at the designated achieve-by point. In the ATD-1 operations, the achieve-by point is always the final approach fix (FAF). ${ }^{3}$

\section{B. Spacing Algorithm History}

The basic goal of an airborne spacing algorithm is to provide a speed, which if flown, will null out the current spacing error. The method used to determine the spacing error and calculate the speed, called the IM speed, is what makes each of the following approaches unique. Starting in the mid-1980's, constant time delay, or time-history, approaches were developed. ${ }^{10,11}$ In these approaches, the spacing error is calculated by determining the time elapsed between the time when the target aircraft passed the IM aircraft's current position and the time when the IM aircraft arrives at that position. The spacing error at any given point is simply the difference between the elapsed time and the assigned spacing goal. Therefore, the IM speed is a combination of the target aircraft's current speed and the speed needed to null the current spacing error. Since the spacing error is based on both aircraft passing over the same position, this type of algorithm can only be used when the aircraft are in-trail.

Starting in the early 2000's, trajectory-based approaches were developed. These algorithms calculate the ETA for each aircraft at the achieve-by point and then compare the difference in ETAs to the assigned spacing goal to determine the current spacing error. The IM speed is then defined as the IM aircraft's expected speed on that segment plus the speed compensation used to null the current spacing error. This type of algorithm relaxes the requirement of the aircraft being in-trail, but requires additional information to calculate the trajectory for both aircraft from their current positions to the common point.

The Eurocontrol-developed algorithm, CoSpace, ${ }^{11}$ limits the time-to-go operation to when both the target and IM aircraft are headed direct to a common point. Therefore, the only information required to construct each of the trajectories is knowledge of the common, or merge, point and current speeds. As this constraint generally limits the operation to ranges of tens of nautical miles (nmi), the uncertainties in the time-to-go are small and manageable over the length of the direct-to leg. The NASA-developed Airborne Spacing for Terminal Arrival Routes (ASTAR) uses detailed route information for both aircraft to allow spacing to begin at any time the target aircraft's route can be communicated to the IM aircraft. ${ }^{12,13}$ This allows for multiple turns, planned altitude changes, and planned speed changes prior to the common point, and for a much larger range between aircraft at the start of the operation. In a mature Next Generation Air Traffic Management (NextGen) environment, the target aircraft's route information would be delivered by a datalink message from the controller. In the interim, however, published RNAV arrival 
routes and instrument approaches can provide sufficiently accurate information for airborne spacing. Since the spacing algorithm is continually running and providing up-to-date speed guidance, any trajectory prediction errors will eventually appear as spacing errors and are corrected.

\section{Air-Ground Integration Challenges}

When the arrival demand exceeds the airport or terminal acceptance rate, aircraft are delayed beyond their nominal arrival time to ensure separation and proper spacing during the arrival to the runway. In the mid-term NextGen environment that ATD-1 is planned for, some of this delay is allocated to, or planned for, the terminal environment with the rest being absorbed between the schedule freeze horizon and the meter fix (the entry to the terminal area). Controllers will use two main pieces of information to inform their decision on how to absorb the delay. The center controllers will be provided with a delay countdown time (DCT), which displays a digital readout of the delay that should be absorbed prior to the meter fix. Terminal controllers will be provided slot markers and associated speeds, which display the location and speed of a trajectory which will absorb the delay allocated to the terminal environment. When possible, controllers will use slower speeds to absorb delay; however, vectoring can be used to lengthen the flight path if speed control is insufficient. Vectoring is generally limited to the center airspace in the ATD-1 environment.

For IM operations that include merging routes, the IM aircraft must be provided with information to build the target aircraft's 4D trajectory. Without datalink communications, the controller includes the name of the target aircraft's RNAV arrival route as part of the verbal clearance. The FIM equipment then accesses an onboard database to extract the necessary information, including the published speed profile. However, the target aircraft has insufficient knowledge of any delay that the target may be required to absorb. This results in ETA calculations which are incorrect by up to a few minutes. The IM Aircraft also tries to maintain the published speed profile, only making correction for spacing errors. Therefore, the IM aircraft may see a small spacing error when it should be large, and as a result, maintain a high speed when the surrounding traffic has slowed. This is seen as undesirable behavior by the en route controllers. The opposite behavior is seen in the terminal area where the IM aircraft is now trying to respond to the delayed time of the target aircraft and can result in particularly slow speeds by the IM aircraft in the terminal area. These slow speeds may not match the speeds of the target aircraft, creating undesirable closure rates when the IM and target aircraft are in-trail.

\section{Spacing Algorithm}

The latest version of the ASTAR algorithm, ASTAR-12, includes modifications to the speed control algorithm that improve performance when the IM aircraft is following a target aircraft with a delayed trajectory. The ASTAR12 algorithm uses proportional control added to a groundspeed feedback term, which calculates the difference between the expected and actual groundspeed of the target aircraft and uses this information to compute the amount of speed compensation required to null the spacing error (Figure 1). The groundspeed feedback acts as a derivative term applied to the portion of the spacing error produced by the target aircraft, enabling the IM aircraft to compensate for errors in the target aircraft's predicted groundspeed and eliminating the undesirable closure rates between the IM and target aircraft.

The ASTAR-12 algorithm incorporates additional features that are designed to improve performance and improve its acceptance by both pilots and controllers. Many of the features were designed to improve the speed control behavior by decreasing the frequency of speed changes.

The first feature, which will be referred to as the groundspeed lockout, disables the groundspeed feedback when the difference between the target's actual and expected groundspeed is positive. The benefit of the groundspeed lockout is a reduced number of commanded speed increases. However, the implementation of the groundspeed lockout also means that target aircraft flying faster than the published profile

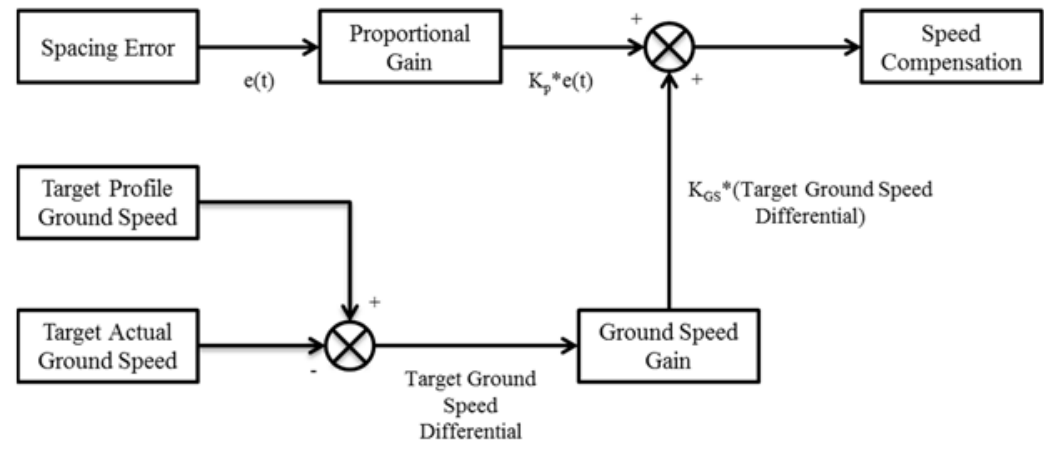

Figure 1. A simplified diagram of the ASTAR-12 speed control algorithm 
speeds can be detrimental to the performance of ASTAR-12. This situation is not expected to happen very often during high demand terminal operations, since aircraft are delayed to eliminate ties at merge points along the arrival.

A second feature is the gain scheduling of the groundspeed feedback term. The gain applied to the target aircraft groundspeed $\left(\mathrm{K}_{\mathrm{GS}}\right)$ is reduced so that it is zero when the IM aircraft is on final approach. This eliminates steady-state error caused by the groundspeed feedback term canceling out the proportional term. One potential disadvantage is that large target speed deviations from the published speed profile when the IM aircraft is on final approach may cause undesirable behavior. However, such speed deviations on final approach are expected to be uncommon occurrences, since the trajectories produced by TMA-TM do not include delay on final approach.

A third feature in ASTAR-12 inhibits speed increases for a certain period of time when a known deceleration point on the arrival is near. This functionality has always been in ASTAR, but the look-ahead time of the function was increased from 20 to 60 seconds. Inhibiting speed increases for a period of time helps prevent speed increases that are shortly followed by speed decreases, also known as speed reversals.

The last feature is a 60 second filter that is applied to the target's groundspeed deviation. Since the groundspeed feedback acts as a derivative term, changes in groundspeed caused by wind error or target aircraft behavior could cause frequent speed changes. This filter smooth's out the target's groundspeed deviation, ensuring that the IM commanded speeds are not affected by noise.

\section{Experiment Design}

\section{A. Scenario Description}

The airspace around Phoenix Sky Harbor International Airport (KPHX) was modeled along with two area navigation (RNAV) arrival procedures for west flow traffic: the EAGUL5 arrival from the northeast and the MAIER5 arrival from the northwest. For the west-flow configuration that was used in this study, the EAGUL5 arrival is the short-side arrival and the MAIER5 arrival is the downwind arrival. All aircraft in the study landed on runway 26, the north-most runway at KPHX. Previous research has shown that the behavior of the original ASTAR algorithm is not dependent on route design; ${ }^{14}$ therefore, only one set of arrival routes was examined.

This simulation investigated 630 pairs of aircraft, consisting of one target aircraft and one IM aircraft. In each of the scenarios, the IM and target aircraft were on different routes to ensure that worse-case wind forecast errors were examined.

\section{B. Experiment Design}

A full factorial experiment was used to investigate the ASTAR-12 algorithm. Two primary independent variables were examined along with several other variables intended to simulate real-life variation. The primary independent variables were seven different target aircraft speed profiles and five different wind error conditions. Both of these variables were expected to excite the groundspeed feedback term that was added to the ASTAR-12 spacing algorithm. Levels for each of these variables were carefully chosen in order to examine the spacing performance of the IM aircraft.

Six different target aircraft speed profiles were designed by subject matter experts to emulate different speed profiles flown by aircraft controlled by ATC (Figure 2). These speed profiles were a nominal profile ("Nom"), where the target aircraft flew the speeds expected by the spacing algorithm; a fast speed profile ("Fast"), where the target maintained a speed higher than the published speeds throughout the arrival; a slow speed profile ("Slow"), where the target aircraft maintained a speed slower than the published speed throughout the arrival; an altitude profile (“Alt”), which added an altitude step-down prior to the terminal airspace to
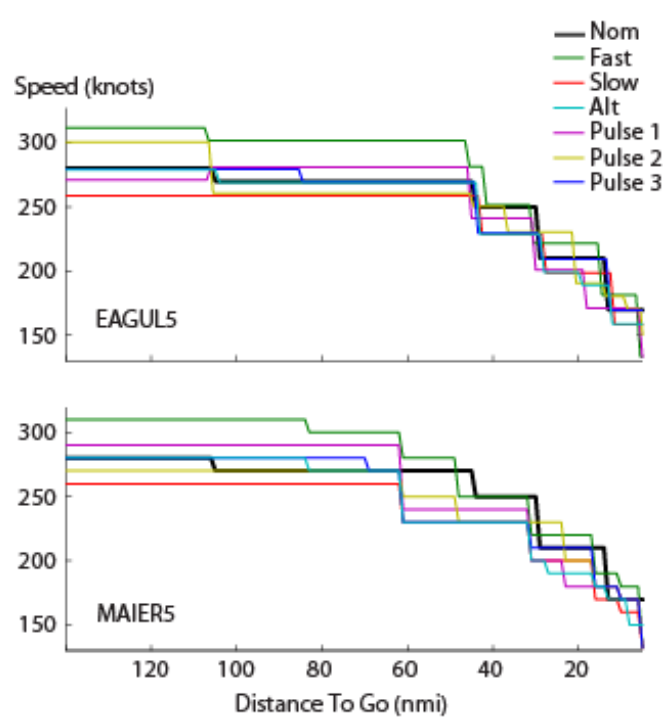

Figure 2: Target speed profiles for each arrival route in the study

the slow speed profile; and three pulse speed profiles which switched between faster than and slower than the published speed profile over the course of the arrival ("Pul 1", "Pul 2", and "Pul 3"). 
The truth and forecast winds used in this study were selected from a set of wind fields observed at Phoenix Sky Harbor Airport in 2011. To conform to current real-world procedures for providing aircraft with descent forecast winds, the IM descent forecast winds were sampled at a single waypoint after top-of-descent at altitudes of 30,000 feet, 24,000 feet, 18,000 feet, and 10,000 feet. Since the IM aircraft is not expected to have access to the target aircraft's descent forecast winds without special accommodations, the IM avionics used the IM aircraft's descent forecast winds to generate 4D-trajectories for both the IM and target aircraft. The ETAs of aircraft flying through the truth and forecast wind fields were compared in order to determine the amount of ETA error caused by the difference between the wind forecast and the actual winds (Figure 3). Wind forecasts were then chosen to meet the following five conditions: target early and IM aircraft early (E/E), target late and IM

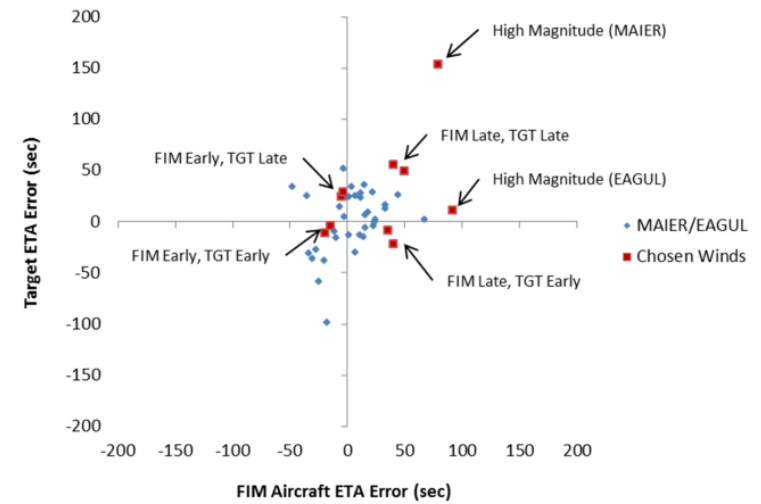

Figure 3: The ETA errors caused by differences between the truth wind fields and the forecast wind fields. The red squares show the winds fields that were used in this study. aircraft late $(\mathrm{L} / \mathrm{L})$, target early and IM aircraft late $(\mathrm{E} / \mathrm{L})$, target late and IM aircraft early (L/E), and a high magnitude wind error (HM). Additionally, the wind fields were selected to keep the ETA errors between the EAGUL5 and MAIER5 arrivals as similar as possible for each of the five wind conditions to retain comparability between arrivals.

Variables of less interest than the target speed profile and wind error were also added to the experimental design. Inclusion of these non-primary variables allowed for examination of the robustness of the algorithm for a variety of circumstances. These additional variables were three different initial spacing errors (-60 sec, $0 \mathrm{sec}, 60 \mathrm{sec}$ ), three different target aircraft weights (light, normal, heavy), and two different target arrival routes (MAIER5 and EAGUL5).

\section{Simulation Platform}

NASA's Airspace and Traffic Operations Simulation (ATOS) platform was used to simulate all of the aircraft in this study. The ATOS platform contains a network of hundreds of real-time simulators that can be used for batch studies and real-time human-in-the-loop experiments. ${ }^{15}$ Each simulated aircraft within the ATOS platform has a high fidelity six degree of freedom dynamics model, an emulated flight management system, and an emulated auto throttle system. The simulated aircraft can either be flown by human pilots or by an automated pilot model that simulates pilot inputs into the aircraft. In this study, all aircraft were flown by the automated pilot model.

\section{Results}

\section{A. Delivery Accuracy and Precision}

The delivery accuracy is the measure of how well the IM aircraft achieved the assigned spacing goal, measured as the difference between the IM and target aircrafts' time of arrival minus the assigned spacing goal at the achieveby point. The design goal is for the mean delivery accuracy to be between \pm 5 seconds and for $99 \%$ of the delivery accuracies to be between \pm 15 second. The mean delivery accuracy was estimated to be 1.76 seconds and t-tests indicated that the mean delivery accuracy was within \pm 5 seconds $(\mathrm{p}<0.001)$. There were fourteen out of the 630 cases where the delivery accuracy was greater than 15 seconds. All fourteen of these observations were associated with a target aircraft flying a fast speed profile in combination with a target aircraft early, IM aircraft late (E/L) wind condition or the high magnitude wind condition (HM), which also caused the target aircraft to arrive early and the IM aircraft to arrive late. Since TMA-TM only delays aircraft to avoid conflicts, cases where target aircraft fly speed profiles substantially faster than the published speeds are not expected to occur often. Therefore, the data was reexamined without the fast target speed profile cases. Without these cases, the mean delivery accuracy dropped to 0.8 seconds, with zero cases having a spacing error greater than fifteen seconds.

To examine factors that may cause changes to the delivery accuracy, a second order regression model was fit to the datasets with and without results involving the fast target speed profile. Main effects and two-factor interactions were found to be statistically significant in both cases; however, the interaction effects were generally small. Additionally, many of the statically significant interactions involved the fast target speed profile. 


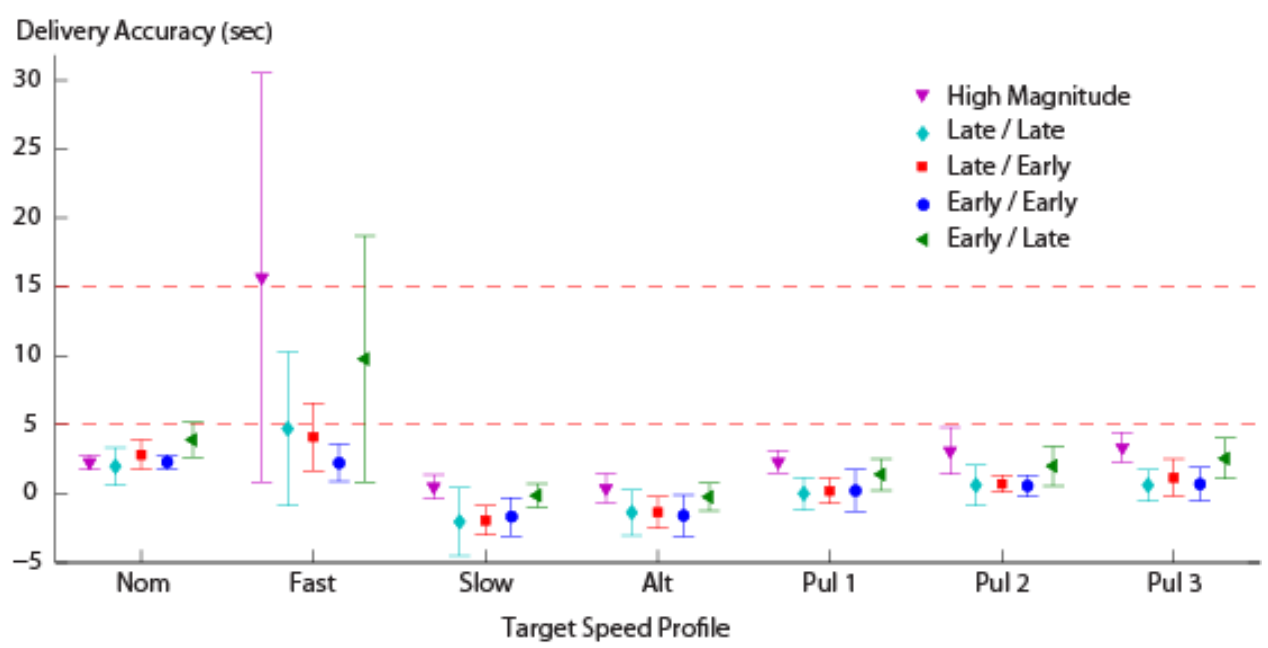

Figure 4: Delivery accuracy and precision by target speed profile and wind condition

In addition, the delivery precision was measured using the standard deviation of the delivery accuracy, which was found to be 4.7 seconds. Though skewed by the presence of the previously mentioned fourteen cases, a Chisquare variance test still shows delivery precision to be within 5 seconds $(\mathrm{p}<0.001)$. Moreover, removing these cases increased the delivery precision to 2.2 seconds.

\section{B. Speed Change Rate}

Within the retrofit implementation of IM that is likely to be used in ATD-1, flight crews will likely be required to close the control loop by entering IM commanded speeds into the mode control panel speed window. As a result, the rate at which new commanded speeds are provided is a measure of pilot task load. The rate of speed changes were computed for three different arrival segments: the Air Route Traffic Control Center (ARTCC) airspace (above 10,000 feet), between entry to Terminal Radar Approach Control (TRACON) and the turn onto final approach, and on final approach.

Table 1: Mean speed change rate and upper $95 \%$ confidence bounds for each flight segment

\begin{tabular}{lccc}
\hline \hline Flight Segment & $\begin{array}{c}\text { Mean Speed Changes } \\
\text { per Minute }\end{array}$ & $\begin{array}{c}\text { Upper 95\% } \\
\text { Confidence Bounds }\end{array}$ & $\begin{array}{c}\text { Planned Procedural } \\
\text { Speed Change Rate }\end{array}$ \\
\hline Center Airspace & 0.29 & 0.30 & 0.06 \\
TRACON & 0.65 & 0.66 & 0.40 \\
Final Approach & 1.28 & 1.30 & 0.70 \\
\hline \hline
\end{tabular}

Previous studies indicated that a speed change rate of less than two speed changes per minute was acceptable to pilots and therefore used as a benchmark for this study. ${ }^{16,17}$ Means and upper 95\% confidence bounds for number of speed commands for each of the segments are shown in Table 1 . The speed change rate includes speed changes used to correct for spacing errors and speed changes that were part of the nominal approach procedures. The portion of the speed change rates that were part of the nominal approach procedures were approximately 0.06 speed changes per minute in the center airspace, 0.4 speed changes per minute in the TRACON, and 0.7 speed changes per minute when on final approach.

On average, the speed change rate increased as the aircraft approached the runway; however, it still remained below the success criteria during the final approach flight segment. To gain further insight into the performance of ASTAR-12, a second order quasi-Poisson regression model was fit for each flight segment. In all three flight segments, both main effects and two-factor interactions were found to be statistically significant; however, further investigation revealed none of the effects to be operationally significant since all speed change frequencies fall below the critical value of two speed changes per minute. 


\section{Spacing Error}

This study investigated an achieve-by IM operation. As such, the ideal behavior is for the algorithm to smoothly null the spacing error by the achieve-by point. The average spacing error along the arrivals for each speed profile and initial condition were computed and are plotted in Figure 5.

Figure 5 shows that the spacing errors converge relatively smoothly to zero before the achieve-by point is reached for all of the conditions tested. The fast speed profiles produced the largest deviations. During the -60 second (early) initial error cases, the spacing error increases past zero and is only nulled at the end of the arrival. During the zero second initial error cases, the spacing error increases past zero to approximately 50 seconds, and is only nulled at the end of the run. During the 60 second (late) initial error cases, the spacing error increases above the other target speed profile spacing errors, and the algorithm appears to have trouble reducing the error to zero near the end of the arrival. This occurs because the groundspeed lockout feature in the ASTAR-12 algorithm turns off the groundspeed feedback term when the target's groundspeed is faster than the expected groundspeed, which causes the spacing algorithm to utilize only proportional control. The small proportional gain when the aircraft is far from the runway does not provide adequate speed compensation to correct for the target aircraft's speed deviation.

\section{Speed Reversals}

One metric of interest was the number of speed reversals in the profile. While some speed reversals may be required to compensate for uncertainty throughout the arrival, a large number of high magnitude speed reversals could indicate that that IM aircraft are not flying the most efficient speeds. Additionally a large number of high magnitude speed reversals could cause human factors concerns, since aircraft speeds normally decrease throughout an arrival.

The number and magnitude of speed reversals was computed by counting the number of consecutive speed increases. For instance, if there were three 10-knot speed increases in a row followed by a speed decrease, one 30knot speed reversal was counted. The average number of speed reversals for each target speed profile and each wind condition are plotted in Figure 6. The gray error bars show the standard deviation of the speed reversals for each condition and the colored segments show the magnitude of the speed reversals.

The results indicate that the high magnitude wind error averaged the largest number of speed reversals, as well as the largest number of high magnitude speed reversals. This is expected, since the high magnitude wind error contained a greater amount of uncertainty than the other wind errors. The slow and altitude target speed profiles had the largest number of speed reversals; however a large majority of these cases were 10-knots or less and therefore not seen as problematic. The pulse 2 target speed profile contained a
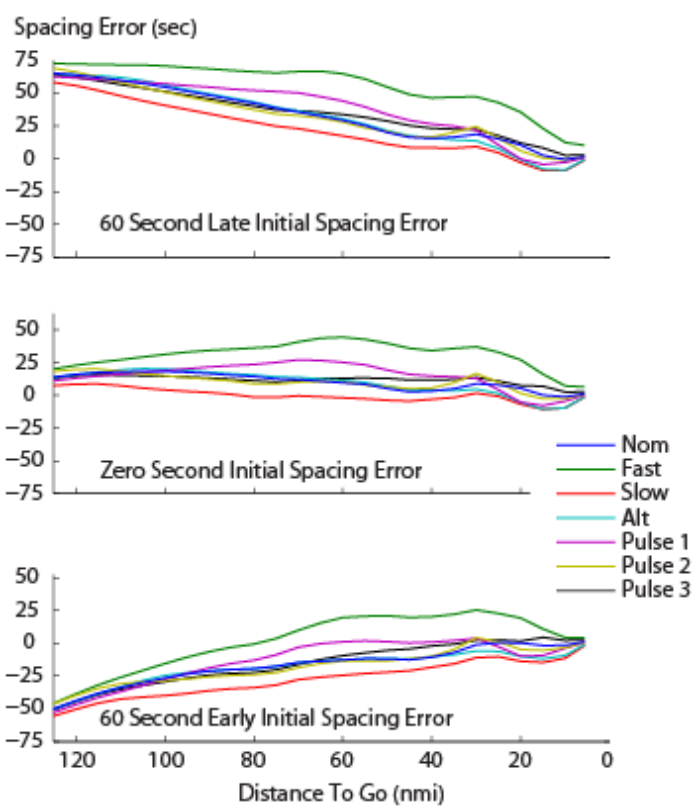

Figure 5: Spacing error along the arrival by initial spacing error
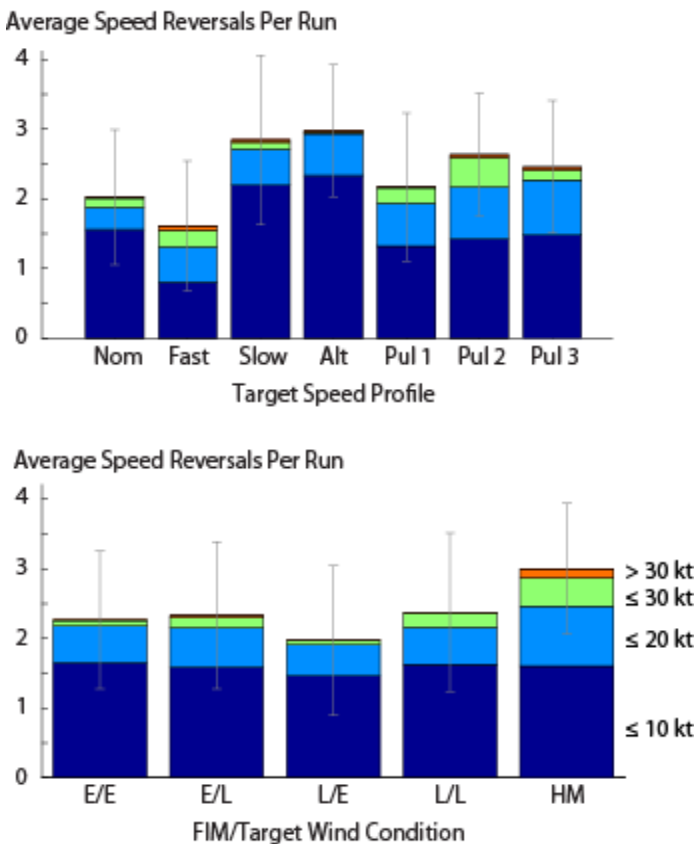

Figure 6: Speed reversals by wind condition and by target speed profile 
substantial number of 10 to 30 -knot speed reversals. It is suspected that a number of the pulse 2 speed reversals occurred because the speed of the target aircraft changed from significantly slower than the nominal profile to faster than the nominal profile.

\section{E. Case Studies}

Generally, the IM aircraft in this study exhibited good performance. The spacing error decreased smoothly, and the aircraft precisely met their assigned spacing goal. However, there were some cases where the groundspeed feedback term caused speed reversals, which may degrade the efficiency of IM operations. Two case studies are described in this section: one with smooth IM speed control behavior and one with several speed reversals.

The first case study is an example where the spacing algorithm smoothly nulled the spacing error while compensating for a delayed target aircraft trajectory (Figure 7). In this case study, the target aircraft flew the slow speed profile and began with a -60 (early) spacing error. The top plot of Figure 7 shows the speeds commanded by the spacing algorithm (blue line) in relation to the expected speed profile (black line). The middle plot shows the target's speeds in relation to the speeds that the spacing algorithm expected. The bottom plot shows the spacing error throughout the arrival. Within this case study, the IM aircraft was commanded a slow IM speed to compensate for the target aircraft's slow speed and the initial negative (early) spacing error. Throughout the arrival, the spacing error smoothly decreased to zero, the IM commanded speed changes were relatively infrequent, and most of the IM speed changes were speed decreases until the IM aircraft was approximately seven nautical miles from the runway. At this point, the spacing error rapidly decreased, and the IM aircraft was commanded by the algorithm to return to the nominal speed profile.

The second case study is from a scenario identical to the first case study, except the target aircraft flew the pulse 2 speed profile instead of a slow speed profile. The pulse 2 speed profile had segments that were above and below the expected speed profile; however, the target's speeds always decreased. Similar to the first case study, the spacing error decreased smoothly to zero by the achieve-by point. However, the IM aircraft's commanded speeds contained several speed reversals. Many of these speed reversals were caused by the target's speed in relation to the expected speed profile. The target aircraft slowed early to absorb delay, creating a target groundspeed deviation and causing the groundspeed feedback term to slow the IM aircraft. When the target aircraft's profile speed slowed to the target's speed its groundspeed deviation returned to zero, causing the speed to increase. As an example of the behavior, consider the speed reversal that occurred when the IM aircraft was between $40 \mathrm{nmi}$ and $30 \mathrm{nmi}$ from the runway. The top plot of Figure 8 shows that the IM aircraft
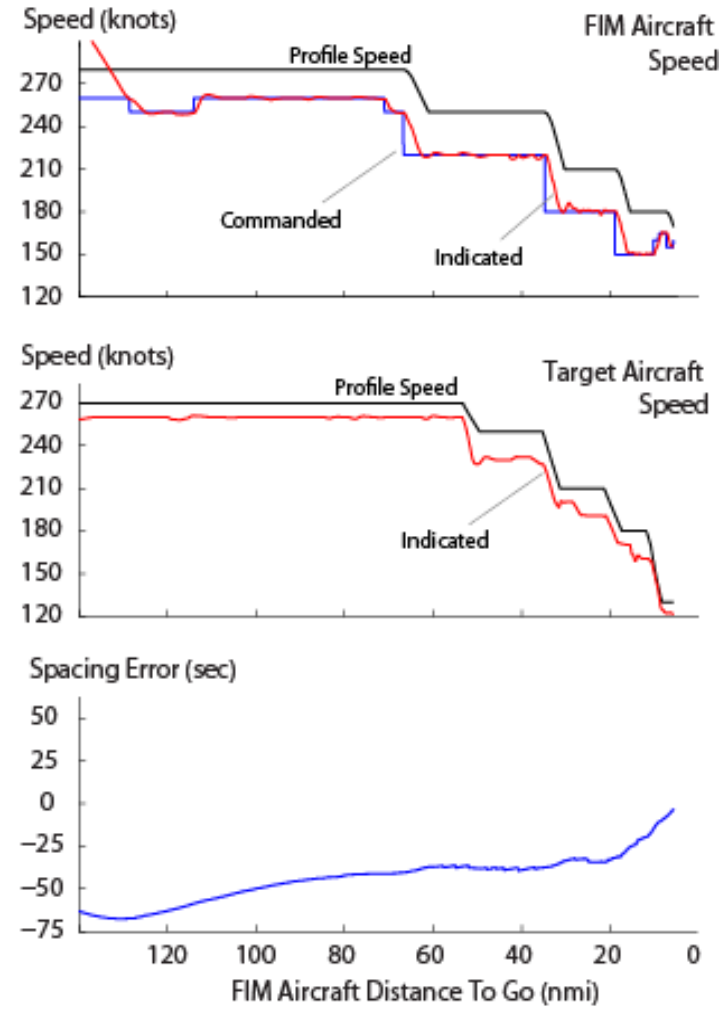

Figure 7: Case Study \#1
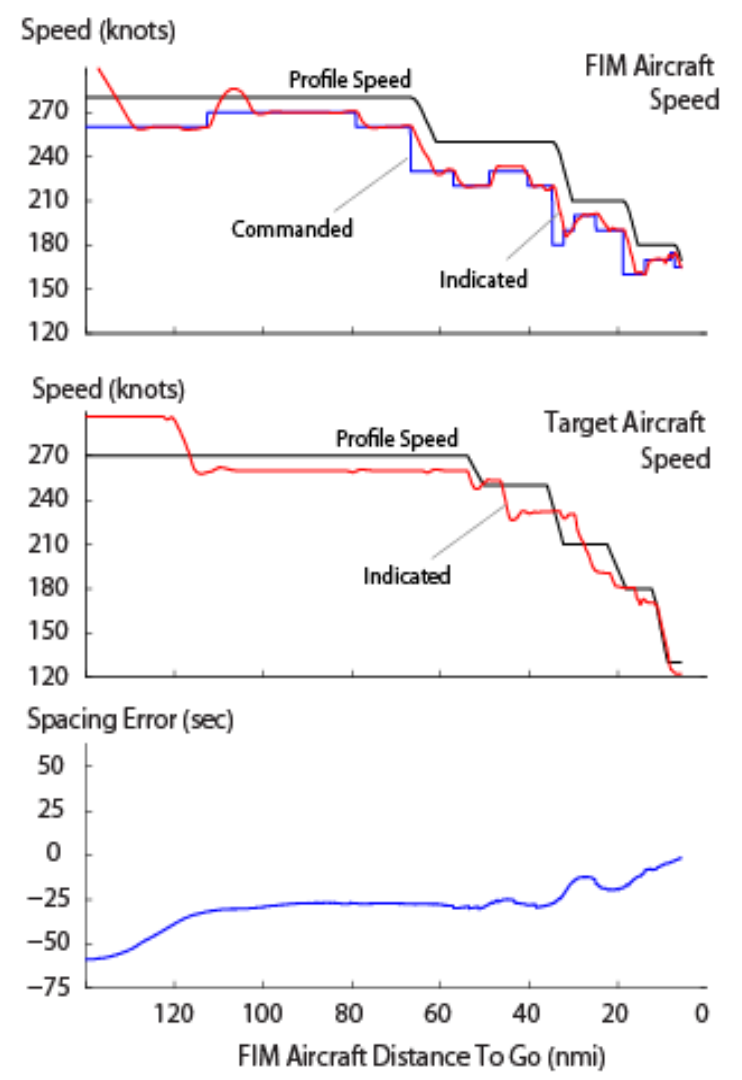

Figure 8: Case Study \#2 
was commanded a large speed decrease, because the published speed decreased. Shortly after, the IM commanded speed increased by 20-knots. The middle plot shows the target's speed changing from 20-knots slower than the expected speeds to 20-knots faster at the same point in time.

The performance of the ASTAR-12 spacing algorithm will depend on how controllers manage their aircraft. If the controllers absorb delay smoothly over the arrival, as was done in the first case study, the algorithm will likely perform well. Conversely, the performance will be reduced if the target's speed deviation from the expected profile varies throughout the arrival.

\section{Conclusion}

This paper presented a study that examined the ASTAR-12 spacing algorithm, which was designed to increase the compatibility between IM and ground scheduling tools by enabling the IM aircraft to follow target aircraft with delayed trajectories that are unknown by the IM avionics. The data from this study demonstrated that the ASTAR-12 algorithm generally exhibited good performance. With the exception of the fast speed profiles, the mean and standard deviation of the inter-arrival spacing at the achieve-by point were less than five seconds, which is consistent with previous IM research. The spacing error was normally nulled smoothly throughout the arrival, and the rate at which speed changes were commanded was typically less than two per minute, which has been found to be acceptable in human-in-the-loop simulations.

In general, ASTAR-12 exhibited good performance; however, two cases were discovered where ASTAR-12 may provide less than desirable performance. The algorithm was not always able to null the spacing error when the target aircraft flew a fast speed profile combined with either a high magnitude wind error or a wind error that caused the target aircraft to arrive late and the IM aircraft to arrive early. Due to the way that aircraft are scheduled, these cases are not expected to occur often in an operational environment; however, they may result in an infeasible IM operation if they do occur. Additionally, the ASTAR-12 algorithm is prone to speed reversals when the target aircraft slows earlier than expected to absorb delay. The magnitude and frequency of these speed reversals will largely depend on the speed commands provided by controllers to the target aircraft.

Further simulation is needed to evaluate the spacing algorithm when there are long strings of aircraft and to evaluate how all of the ATD-1 technologies perform when used by pilots and controllers. One aspect of this work that should be investigated is the interaction between an IM aircraft and surrounding non-IM aircraft when the IM aircraft is following a target aircraft on a different route.

\section{Acknowledgements}

The authors would like to thank the members of the ATOS software development team and the ATOL operations staff who invested countless hours of hard work. This experiment and this paper would not be possible without their dedication.

\section{References}

${ }^{1}$ R. Babbitt, "FAA Aeropace Forecast: Fiscal Years 2011-2031," US Department of Transportation Federal Aviation Administration Aviation Policy and Plans, pp. 2011-2031, 2010.

${ }^{2}$ J. Robinson and M. Kamgarpour, "Benefits of Continuous Descent Operations in High-density Terinal Airspace Under Scheduling Constraints," in 10th AIAA Aviation Technology, Integration, and Operations (ATIO) Conference, Fort Worth, 2010.

${ }^{3}$ B. T. Baxley, H. N. Swenson, T. Prevot and T. J. Callantine, "NASA's ATM Technology Demonstration-1: Integrated Concept of Arrival Operations," in Digital Avionics Systems Conference (DASC), 2012 IEEE/AIAA 31st, 2012.

${ }^{4}$ D. Isaacson, J. Robinson III, H. Swenson and D. Denery, "A Concept for Robust, High-Density Terminal Air Traffic Operations," in 10th AIAA ATIO Conference, Fort Worth, 2010.

${ }^{5}$ H. Swenson, J. Thipphavong, A. Sadovsky, L. Chen, C. Sullivan and L. Martin, "Design and Evaluation of the Terminal Aera Precision Scheduling and Spacing System," in Ninth USA/Europe Air Traffic Management Research \& Development Seminar, Berlin, 2011.

${ }^{6}$ T. Callantine, E. Palmer and M. Kupfer, "Human-in-the-loop Simulation of Trajectory-based Terminal-area Operations," in 27th International Congress of the Aeronautical Sciences (ICAS), Nice, 2010.

${ }^{7}$ M. Kupfer, T. Callantine, L. Martin, J. Mercer and E. Palmer, "Controller Support Tools for Schedule-based Terminalarea Operations," in Ninth USA/Europe Air Traffic Management Research and Development Seminar, Berlin, 2011.

${ }^{8}$ B. E. Barmore, T. S. Abbott, W. R. Capron and B. T. Baxley, "Simulation Results for Airborne Precision Spacing along Continuous Descent Arrivals," Perspective, vol. 22, p. 27, 2008.

${ }^{9}$ J. L. Murdoch, B. E. Barmore, B. T. Baxley, W. R. Capron and T. S. Abbott, "Evaluation of an Airborne Spacing Concept to Support Continuous Descent Arrival Operations," in Eighth USA/Europe Air Traffic Management Research and Development Seminar, Hampton, 2009. 
10 J. R. Kelly and T. S. Abbott, "In-trail spacing dynamics of multiple CDTI-equipped aircraft queues," National Aeronautics and Space Administration, Hampton, 1984.

${ }^{11}$ E. Hoffman, D. Ivanescu, C. Shaw and K. Zeghal, "Analysis of Constant Time Delay Airborne Spacing Between Aircraft of Mixed Types in Varying Wind Conditions," in Fifth USA/Europe Air Traffic Management R\&D Seminar, Budapest, 2003.

12 B. Barmore, T. Abbott and K. Krishnamurthy, "Airborne-Managed Spacing in Multiple Arrival Streams," in 24th International Congress of the Aeronautical Sciences, Stockholm, 2004.

${ }^{13}$ T. Abbott, "An Overview of a Trajectory-Based Solution for En Route and Terminal Self-Spacing: Fourth Revision," National Aeronautics and Space Administration, Hampton, 2013.

${ }^{14}$ B. E. Barmore, T. S. Abbott and W. R. Capron, "Evaluation of Airborne Precision spacing in a Human-in-the-Loop Experiment," in AIAA 5th Aviation Technology, Integration, and Operations Conference (ATIO), Reston, VA, 2005.

${ }^{15}$ M. E. Peters, M. G. Ballin and J. S. Sakosky, "A Multi-Operator Simulation for Investigation of Distributed Air Traffic Management Concepts," in AIAA Modeling and Simulation Technologies conference and Exhibit, August 5-8, Monterey, California, 2002.

${ }^{16}$ C. Hebraud, E. Hoffman, N. Pene, L. Rognin and K. Zeghal, "Assessing the impact of a new air traffic control instruction on flight crew activity," in AIAA Guidance, Navigation, and Control Conference, Providence, Rhode Island, 2004.

${ }^{17}$ E. Hoffman, N. Pene, L. Rognin and K. Zeghal, "Introducing a new spacing instruction. Impact of spacing tolerance on flight crew activity," in Proceedings of the Human Factors and Ergonomics Society Annual Meeting, 2003. 